\title{
LynxNet: Wild Animal Monitoring Using Sensor Networks
}

\author{
Reinholds Zviedris ${ }^{1}$, Atis Elsts ${ }^{12}$, Girts Strazdins ${ }^{12}$, \\ Artis Mednis ${ }^{12}$, and Leo Selavo ${ }^{12}$ \\ 1 Faculty of Computing, University of Latvia, \\ 19 Raina Blvd., Riga, LV 1586, Latvia \\ 2 Institute of Electronics and Computer Science, \\ 14 Dzerbenes Str, Riga, LV 1006, Latvia \\ reinholds@zviedris.com, \{aelsts, gstrazdins, selavo\}@acm.org, \\ artis.mednis@edi.lv
}

\begin{abstract}
Monitoring wild animals, especially those that are becoming endangered (for example, lynxes and wolves) is important for biology researchers. Solutions for the monitoring already exist; however, they all have drawbacks, such as limited range or lifetime, sensing modality, reporting delays, unreliability of operation. In this work we describe our experiences in designing an improved animal monitoring sensor system and low-level software for sensor node control and communication. The target animals for this particular research are wild lynxes or canines, however it can be extended to other animal species. The LynxNet system is based on tracking collars, built around TMote Mini sensor nodes, sensors, GPS and 433MHz radio, and stationary base stations, placed at the locations that are frequented by the animals. We present preliminary field results of our radio communication range tests.
\end{abstract}

Key words: Animal monitoring, Low power sensing, Sensor networks, Delay-tolerant networks

\section{Introduction}

Monitoring the wild animal behavior and whereabouts is a challenge because the animals avoid human beings. The commercially available solutions provide monitoring devices that have limited sensing capabilities, communications requiring cellular coverage or have long data report delays [1]. We propose LynxNet system with extended sensing modality and multi hop delay tolerant communication approach. Our collaborators - biology scientists[3] aim to track Eurasian lynx (Lynx lynx) migration in Latvian forests. Our challenge is to achieve long-term operation with a single set of batteries in the forest environment with no energy harvesting. Our contribution includes design of simple yet persistent animal monitoring architecture for resource-constrained mobile sensor systems, development of efficient PHY and MAC layer radio communication protocols and analysis of radio communication range in field tests.

This work has been partially supported by ESF under grants Nr. 2009/0219/1DP/ 1.1.1.2.0/APIA/VIAA/020 and Nr. 2009/0138/1DP/1.1.2.1.2/09/IPIA/VIAA/004. 


\section{Related work}

A number of animal monitoring sensor systems have been developed in the past few years [7][6][2][4]. The most common to our hardware is ZebraNet [7] animal tracking collar. However, not enough solar energy is available for harvesting, and lynx is smaller animal than a zebra requiring a more compact and lightweight solution.

Commercial products for GPS-based tracking are available, such as Tellus collars[1]. In comparison, LynxNet employs a wide modality of sensors in addition to GPS location, that also provide data about the surrounding environment and help to detect patterns of activities of the animal.

\section{System Architecture}

LynxNet is mobile sensing and sparse radio connectivity network (see Figure 1). The architecture offers animal-centric paradigm for sensing at the edge of the Internet using an opportunistic sensor networking approach.

LynxNet nodes are producing two types of packets. First type contains GPS location and fix quality information, temperature, relative humidity and amount of ambient light. One packet is formed once every hour. Second type packet contains data from 3D accelerometer and 2D gyroscope that can be used to calculate motion vector. Every 5 minutes 5 samples of data are gathered, stored in 5 packets to help with the lynx activity classification.

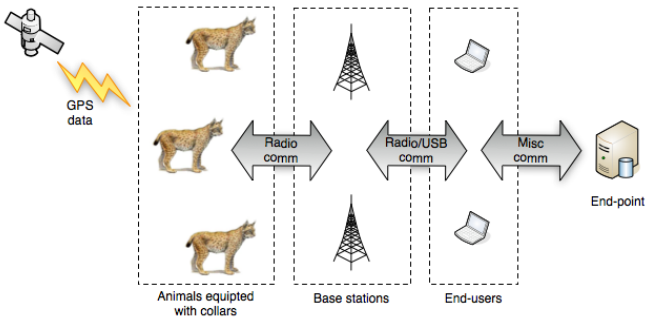

Fig. 1: LynxNet system architecture.

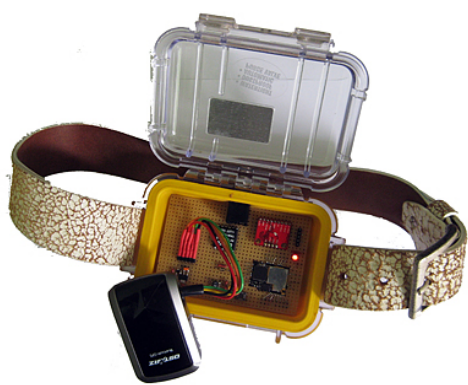

Fig. 2: LynxNet collar device.

\subsection{Hardware}

LynxNet system hardware is organized as three tiers of devices: the animal collar devices (see Figure 2), the base station devices and the client devices. All devices use TMote Mini sensor nodes with TI MSP430F1611 micro-controller. Additionally LINX TRM-433-LT 433MHz transceiver (TRM) has been chosen due to its long-range characteristics.

The collar device has a radio antenna with annular operation directional diagram, GPS receiver and sensors. The prototype uses 1100 mAh 3.7V Li-Poly battery.

The base station uses four stacked half-wave dipole collinear antennas. 


\subsection{Software}

All devices were programmed using MansOS [5] operating system for networked embedded devices developed at University of Latvia. MansOS supports unix-like abstractions, programming in $\mathrm{C}$ and easy portability to new hardware platforms. LynxNet software extends MansOS with API for flash memory access, humidity and light sensor drivers, GPS readings parser, DCO recalibration support, the TRM chip driver, and a design of a MAC-layer communication protocol.

One of the most labor-intensive parts in the project was the development of the transceiver chip driver and PHY level communication protocol. The TRM chip has high receive sensitivity and supports symbol rates up to $10 \mathrm{kBdps}$, but provides only the simplest programming interface, and does not come with any software libraries or sample code. The chip uses OOK modulation, suitable for our project, because interference and high background noise levels are not likely in remote areas, where the system will be deployed. We implemented a packet encoding and decoding algorithm based on Manchester encoding (ME). Using ME decreases the maximal date rate to $5000 \mathrm{bps}$, but allows to decode frames with fewer errors.

On top of the physical level communication, a design for MAC protocol is build. We are going to use a CSMA-based MAC - this method is proven to work well in sparse networks where collision probability is small. The MAC protocol works differently on base stations, which are continuously listening, and mobile nodes, which have low duty cycle. All communication is initiated by the mobile nodes, which periodically poll for nearby base stations.

\section{Evaluation}

The LynxNet hardware field tests were performed in two locations: Rumbula airfield and Sampeteris forest, Latvia. A dog was used instead of a lynx for the better control. The animal, equipped with LynxNet collar device, was moving away from the base station. The amount of received packets was measured after every 50 meters. The collar device was sending out 22 byte packets as in real deployment situation using encapsulation described in Section 3.2.

First, we tested the hardware with TRM radio and then for comparison with CC2420 radio. Once the TRM reception tests started to fail due to the distance, we attached headphones to the base station device and listened for the carrier (beep) signal.

The results of the tests are shown in Table 1. At the distance of 300 meters we received no more packets but were still able to hear the beeping sound for up to 500 meters in the line of sight. In comparison, CC2420 tests received packets up to 165 meters. The forest base station antenna was attached to a tree. At the distance of 300 meters we stopped receiving the packets, but were still able to hear the carrier signal up to 350 meters.

TRM tests showed, that our collar antenna has a significant directional behavior. The best reception was achieved when the animal was standing with a side towards the base station. As expected, at $433 \mathrm{MHz}$ frequency the radio was less affected by the obstacles than at $2.4 \mathrm{GHz}$. 
Table 1: Radio field test results

Table 2: Collar energy consumption

\begin{tabular}{|c|c|c|c|c|c|c|c|c|}
\hline \multirow{3}{*}{$\begin{array}{c}\text { Distance } \\
(\mathrm{m})\end{array}$} & \multirow{2}{*}{\multicolumn{2}{|c|}{$\begin{array}{l}\% \text { of packets } \\
\text { received }\end{array}$}} & \multirow{2}{*}{\multicolumn{2}{|c|}{$\begin{array}{c}\text { RSSI } \\
(\max =4095)\end{array}$}} & \multirow[t]{2}{*}{ Mode } & \multicolumn{2}{|c|}{ Active } & \multirow{2}{*}{$\begin{array}{l}\mathrm{mW} \\
\text { daily }\end{array}$} \\
\hline & & & & & & sec/hour & $\mathbf{m W}$ & \\
\hline & Airfield & Forest & Airfield & Forest & Sleep & 3527.123 & 0.033 & 0.78 \\
\hline 50 & $80-100 \%$ & $80-100 \%$ & 2700 & $2800-3000$ & GPS & 60.0 & 218.79 & 87.52 \\
\hline 100 & $80 \%$ & $80-100 \%$ & 2200 & $2200-2500$ & Sensors & 12.0 & 37.62 & 3.01 \\
\hline 150 & $80 \%$ & $70-90 \%$ & $1900-2000$ & 2300 & Radio RX & 0.246 & 31.68 & 0.05 \\
\hline 200 & $40-80 \%$ & $10-50 \%$ & $1600-1800$ & $1600-1800$ & Radio TX & 0.631 & 52.14 & 0.22 \\
\hline 250 & $10-80 \%$ & $20-50 \%$ & $1600-1800$ & $1600-1800$ & & & Total: & 91.57 \\
\hline
\end{tabular}

We also measured energy consumption by the collar device and estimated savings with respect to the duty cycle as seen in Table 2. Based on this information and the current configuration of the prototype the lifetime is 1.5 months. Changing the GPS to MN5010HS and using a battery with greater capacity such as Enix Energies 800040 of $6800 \mathrm{mAh} 3.75 \mathrm{~V}$ the lifetime would be extended up to 15 months.

\section{Conclusion and Future Work}

In this paper, we have presented our experiences designing LynxNet - an animal monitoring system in the wild. We have created a hardware prototype of a highly mobile, energy-efficient monitoring system that gathers accurate GPS position and multimodal sensor data and disseminates it through the system of delay tolerant network nodes to the consumer. Our field tests show that radio communication range of $200-250 \mathrm{~m}$ is achievable and should be considered in further system design.

The future work includes further evaluation of collar device after longer deployments, selection of optimal components and robust packaging design.

\section{References}

1. Followit Wildlife, http://www.followit.se/wildlife/

2. GPS GSM lynx tracking in the Bavarian Forest National Park, http: //www .environmental-studies.de/projects/24/GPS-lynx-tracking/ gps-lynx-tracking.html

3. Latvian State Forest Research Institute "Silava", http://www.silava.1v

4. POST - Pacific Ocean Shelf Tracking Project, http://postcoml.org/

5. Strazdins, G., Elsts, A., Selavo, L.: MansOS: Easy to Use, Portable and Resource Efficient Operating System for Networked Embedded Devices. In: Proc. SenSys'10 (2010)

6. Wark, T., Crossman, C., Hu, W., et al.: The design and evaluation of a mobile sensor/actuator network for autonomous animal control. In: Proc. IPSN'07. pp. 206-215 (2007)

7. Zhang, P., Sadler, C., Lyon, S., Martonosi, M.: Hardware design experiences in ZebraNet. In: Proc. SenSys'04. pp. 227-238 (2004)

The original publication is available at www.springerlink.com: http://www . springerlink. com/content/573467t6gw8q3146/ 